\title{
LA AZOOSPERMIA NO DEBERÍA OFRECERSE COMO RESULTADO DE LA VASECTOMÍA.
}

\author{
Osvaldo Rajmil, Marlin Fernández, César Rojas-Cruz, Carlota Sevilla', Mireia Musquera’ y \\ Eduardo Ruiz-Castañe.
}

Servicio de Andrología y Servicio de Urología'. Fundació Puigvert. Barcelona. España.

\begin{abstract}
Resumen.- OBJETIVO: La vasectomía es un método quirúrgico de contracepción masculina. Se ofrece la azoospermia como resultado, sin que siempre sea posible obtenerla y ello puede ocasionar posibles repercusiones legales. El propósito de este estudio es conocer el número de seminogramas necesarios para conseguir la azoospermia después de la intervención.
\end{abstract}

MÉTODOS: Análisis retrospectivo de hombres a los cuales se les realizó la vasectomía durante un período de 15 meses y con un seguimiento posterior de 2 años. Se realizaron seminogramas consecutivos, más de cinco, con intervalos de 2 a 3 meses en cada uno de los que se obtenía persistencia de espermatozoides.

RESULTADOS: Se intervinieron 618 hombres, 106 fueron descartados por no aportar muestras de semen (17\%) y $2(0.39 \%)$ por presentar espermatozoides móviles, considerándose fallo de la técnica. 510 siguieron el control completo. $316(61.9 \%$ resultaron azoospérmicos en el primer seminograma, 74 (14.5\%) en el segundo, 27 $(5.2 \%)$ en el tercero, $6(1.2 \%)$ en el cuarto y $1(0.2 \%)$

$\frac{0}{0}$
$\frac{0}{0}$
$\frac{0}{0}$
$\frac{0}{0}$
$\frac{0}{0}$
0
0
0

Osvaldo Rajmil

Servicio de Andrología

Fundació Puigvert

Cartagena, 340-350

08025. Barcelona. (España)

orajmil@fundacio-puigvert.es

Trabajo recibido: 23 de junio 2006 en el quinto seminograma. Los 86 (16.8\%) restantes siguieron presentando espermatozoides en el eyaculado más allá del quinto exámen seminal. Los espermatozoides persistentes fueron todos inmóviles e inferiores a $100,000 / \mathrm{ml}$. No se reportaron casos de embarazos en los dos años de seguimiento.

CONCLUSIONES: El número de análisis post quirúrgico puede ser mayor de cinco. La presencia de espermatozoides inmóviles en el eyaculado despues de la vasectomía es frecuente, incluso despues de largos períodos posteriores a la cirugía. Recuentos espermáticos iguales o inferiores a 100,000/ml e inmóviles deberían considerarse aceptables como objetivo del método. Se debería informar acerca de la posible persistencia de espermatozoides inmóviles en el semen. En el consentimiento no debería constar la azoospermia como resultado de la intervención ya que es frecuente encontrar espermatozoides inmóviles y ser igualmente aceptable. Al igual que otros, se debe ofrecer la vasectomía como otros métodos contraceptivos eficaz, pero con posibilidad de fallos.

Palabras clave: Vasectomía. Azoospermia. Contracepción. Embarazo. Semen. Legal. Índice de Pearl.

Summary.- OBJECTIVES: Vasectomy is a surgical method of male contraception. Azoospermia is offered as result of the technique and this is not always attained, resulting in legal matters. The purpose of this study is to know the number of semen samples needed to discharge a patient after intervention. To identify sperm count on semen analysis at time of discharge.

METHODS: Retrospective study of men who underwent vasectomy in a 15-month period with a 2 year follow up. Consecutive semen analyses up to 5 samples were 
measured at 2 to 3 months interval in all men who had persistence of spermatozoa.

RESULTS: 618 men were intervened, 106 did not bring semen to the laboratory (17\%), $2(0.39 \%)$ presented motile sperm and were considered a failure of the technique and excluded. 510 men completed controls. $316(61.9 \%)$ were azoospermic in the first sperm analysis, $74(14,5 \%)$ in the second, $27(5,2 \%)$ in the third, $6(1$, $2 \%)$ in the fourth and one $(0,2 \%)$ in the fitth analysis. The remaining 86 men (16.8\%) had persistence of immotile sperm in the ejaculate and were less than $100,000 / \mathrm{ml}$. No pregnancy was reported during 2 years follow up or after.

CONCLUSIONS: Five or more semen analysis can be made after the surgery. Persistence of immotile sperm in the ejaculate is frequent and may exist for a long period afterwards. Immotile sperm count of $100.000 / \mathrm{ml}$ or less should be accepted as result of the procedure. The patient should be informed about the fact that persistent immotile sperm can be found in his semen. In the informed consent azoospermia should not be a concern as it is frequent to find immotile sperm in the ejaculate and this is an acceptable issue. As with other contraceptive methods, vasectomy should be offered as a safe method although clearly stating that the possibilities of failure do exist

Keywords: Vasectomy. Azoospermia. Birth control. Pregnancy. Semen. Legal. Pearl index.

\section{INTRODUCCIÓN}

La vasectomía es un método anticonceptivo masculino $(1,2)$. Es un método quirúrgico, de barrera, ya que impide el paso de los espermatozoides a través del conducto deferente (3). Los resultados son frecuentemente medidos por la ausencia de espermatozoides (azoospermia) en el eyaculado y este hallazgo ha sido empleado como un indicador del éxito de la cirugía. Este criterio no siempre se logra observándose persistencia (4) de espermatozoides inmóviles.

Otro motivo frecuente de controversia se centra en decidir cuál es el número de seminogramas que deben realizarse en los controles posteriores a la cirugía (5-8) y cuando hay persistencia de espermatozoides, se produce un conflicto en relación a lo ofertado antes del procedimiento quirúrgico (9). Por ello, es importante que la información previa que se brinda al paciente sea correcta para evitar reclamaciones y demandas legales $(10,11)$. Lógicamente, también es importante, de igual forma que ocurre con otros métodos anticonceptivos, informar acerca de la posibilidad de fallo en la técnica quirúrgica (12).
Se requiere una explicación clara en el preoperatorio e informar: que los resultados del procedimiento serán posteriormente evaluados mediante análisis de semen; que el propósito es conseguir un eyaculado libre de espermatozoides y que, aún hombres que resultan azoospérmicos post-vasectomía, pueden producir gestaciones en sus parejas $(12,13)$. Se sabe que el tiempo requerido para que sea evidente la desaparición de espermatozoides es variable y no predecible (14).

Presentamos un estudio observacional, retrospectivo de una población sometida a vasectomía como método de contracepción, en el cual se efectuó un seguimiento de los seminogramas y se registraron la ocurrencia de embarazos durante un período de dos años.

\section{OBJETIVOS}

Evaluar el número de seminogramas necesarios para dar el alta al paciente después de la intervención y las características seminales (recuento) espermático en momento de dar el alta.

\section{MATERIAL Y MÉTODOS}

Se estudiaron de forma retrospectiva a los hombres sometidos a vasectomía durante un período de 15 meses comprendido entre noviembre del 2001 a enero del año 2003. Todos ellos con un seguimiento posterior de 2 años.

Se realizaron seminogramas consecutivos, más de cinco, con intervalos de 2 a 3 meses entre cada uno de ellos. Los análisis y controles se repitieron en los que se objetivaba persistencia de espermatozoides. Los pacientes obtenían la muestra por masturbación y la remitían al laboratorio para estudio (15). La técnica habitual para procesarla en el laboratorio de nuestro centro consiste en observar al microscopio y, en caso de ausencia de espermatozoides, centrifugar a $1.500 \mathrm{rpm}$. El botón residual se re-suspende en $0.5 \mathrm{ml}$ de plasma seminal para volver a ser revisado bajo microscopio (16). En caso de persistencia de espermatozoides, se emplea una cámara de Neubauer para realizar el recuento. Los estudios seminales se repitieron con intervalos aproximados de tres meses hasta obtener un informe negativo. En cada uno de los pacientes se registró el número de seminogramas necesarios para conseguir la azoospermia o en caso de persistencia, el número de espermatozoides inmóviles por $\mathrm{ml}$ en el eyaculado. 


\section{RESULTADOS}

De 618 hombres intervenidos durante el período establecido, 106 fueron descartados del estudio por no acudir a los controles posteriores (17.15\%). Dos casos fueron excluidos por persistencia de espermatozoides móviles $(0.39 \%)$, considerándose fallo en la técnica quirúrgica. Los restantes 510 (Ver Tabla 1) hombres completaron el estudio. La edad media de la población estudiada fue de 42 años, con un rango (27-59). Los hombres que tenían persistencia después del primer análisis presentaron un recuento de espermatozoides inferior a $100,000 / \mathrm{ml}$ o menos y en todos los casos resultaron inmóviles. 86 individuos (16.8\%) continuaron con espermatozoides en el eyaculado después de más de 5 análisis y no reportaron embarazos durante el tiempo de seguimiento.

\section{DISCUSIÓN}

En este estudio mas de un $60 \%$ de hombres a quienes se le realizó la vasectomía como método de anticoncepción, fueron dados de alta con azoopermia en el primer seminograma, un $20 \%$ presentaron espermatozoides inmóviles hasta el quinto seminograma y algo más de un $16 \%$ presentaron espermatozoides inmóviles después del quinto análisis. Esto equivale a más de un año de seguimiento por parte del laboratorio y visitas para evaluar la cirugía.

Nosotros, al igual que otros autores (17-19), hemos considerado que la presencia de espermatozoides móviles es indicativa de fallo de la técnica $(0.4 \%)$ y de la necesidad de reintervención. La persistencia de espermatozoides inmóviles en el eyacu- lado en un alto porcentaje de los hombres estudiados demuestra la necesidad de cambiar la información dada a los pacientes en el pre-operatorio sin que ello signifique la no consecución del objetivo para el cual se realizó la cirugía.

Como otros autores $(20,21)$, podemos concluir que la presencia de espermatozoides inmóviles en el eyaculado no es indicación para repetir nuevos seminogramas ya que con recuentos espermáticos iguales o inferiores a $100.000 / \mathrm{ml}$ no hemos recibido comunicación de embarazos. Consideramos pues, que este puede ser el número de corte aceptable para no realizar más controles y que no se debe esperar la azoospermia para dar el alta a los pacientes. Así debería quedar expresado en el documento de autorización quirúrgica que el paciente firma antes de la intervención.

La vasectomía debería ofrecerse como un anticonceptivo seguro (22), como otros femeninos y masculinos. Cuando se considere su seguridad-efectividad, se debería comparar con la que ofrece la ligadura de trompas en la mujer. Considerar el empleo del índice de Pearl (23) para valorar su eficacia de igual manera que con la de otros contraceptivos. El análisis de semen es un elemento de valoración de la barrera quirúrgica creada. Los médicos, cuando ofrecemos este método, estamos proponiendo una técnica quirúrgica altamente eficaz y debemos asumir que puede eliminar, aunque no totalmente, los espermatozoides del eyaculado. Hay que aceptar el concepto de que la vasectomía tiene como objetivo obtener un semen que, aun conteniendo espermatozoides inmóviles, no mantiene su capacidad fecundante normal (24). Los pacientes pueden ser dados

TABLA I. AZOOSPERMIA PROGRESIVA EN CONTROLES DE SEMEN POST VASECTOMÍA

\begin{tabular}{|c|c|c|c|c|c|c|}
\hline № de hombres & $\begin{array}{c}\text { Azoospermia } \\
1^{\text {er }} \text { control }\end{array}$ & $\begin{array}{c}\text { Azoospermia } \\
2 \text { control }\end{array}$ & $\begin{array}{c}\text { Azoospermia } \\
3^{\text {er }} \text { control }\end{array}$ & $\begin{array}{c}\text { Azoospermia } \\
4^{\circ} \text { control }\end{array}$ & $\begin{array}{c}\text { Azoospermia } \\
\text { 5 control }\end{array}$ & $\begin{array}{c}\text { Persistencia en } \\
\text { posteriores }\end{array}$ \\
\hline 316 & $61.9 \%$ & & & & & \\
\hline 74 & & $14.5 \%$ & & & & \\
\hline 27 & & & $5.2 \%$ & & & \\
\hline 6 & & & & $1.2 \%$ & & \\
\hline 1 & & & & & $0.2 \%$ & \\
\hline 86 & & & & & & $16.8 \%$ \\
\hline
\end{tabular}


de alta después del primer seminograma si tienen un recuento igual o inferior a $100.000 / \mathrm{ml}$. Es importante recordar también que la posibilidad de embarazo existe aún cuando se haya confirmado la azoospermia en el seminograma. (25-27).

\section{CONCLUSIÓN}

Es frecuente encontrar la presencia de espermatozoides inmóviles en el seminograma aun después de un largo periodo posterior a la vasectomía. Un recuento espermático de 100.000/ml e inmóviles, debe considerarse seguro para dar el alta y descontinuar el seguimiento. Se debe informar a los pacientes que existe la posibilidad de hallar espermatozoides inmóviles en el semen en un $40 \%$ aproximadamente después de la primera prueba. De que aún así la posibilidad de embarazo es muy baja. El cambio en los criterios y en la información, tendría repercusiones en el aspecto legal.

\section{BIBLIOGRAFÍA y LECTURAS RECOMENDADAS (*lectura de interés $y^{* *}$ lectura fundamental)}

*1. RAJFER, J.; BENNETT, C.J.: "Vasectomy". Urol. Clin. North Am.; 15: 631-4. 1988.

*2. HANCOK, P.; MCLAUGHLIN, E.: "British Andrology Society guidelines for the assessment of Post Vasectomy Semen Samples". J. Clin. Pathol. 2002; 55: 812-816. 2002.

3. LABRECQUE, M.; PILE, D.; SOKAL, D. y cols.: "Vasectomy surgical techniques in South and South East Asia". BMC Urology;5:10. 2005.

4. SIVARDEEN, K.A.; BUDHOO, M.: "Post vasectomy analysis: Call for a uniform evidence based protocol". Ann. R. Coll. Sur. Engl. May; 83:177-9. 2001.

5. ADMUNDSEN, G.A.; RAMAKRISHNAN, K. "Vasectomy: A "Seminal"Analysis". South Med. J.; 97: 54-60. 2004.

6. CHAWLA, A.; BOWLES, B.; ZINI, A.: "Vasectomy follow-up: clinical significance of rare nonmotile sperm in postoperative semen analysis". J. Urol.; 6: 1212-5. 2004.

7. SMITH, A.G.; CROOKS, J.; SINGH, N.P.: "Is the timing of post-vasectomy seminal analysis important". Br. J. Urol.; 81: 458-60. 1998.

8. BRADRAKUMAR, C.; GOGOI, N.K.; SUNDARAM, S.K.: "Semen Analysis after vasectomy: when and how many". BJU Int.; 86: 479-81. 2000.

9. DE KNIJFF, D.W.; VRIJHOFHJ, ARENDS, J. y cols.: "Persistence or reappearance of nonmotile sperm alters vasectomy: Does it have clinical consequences”. Fert Steril; 67: 332. 1997.

10. ARANGO TORO, O.; ANDOLZ PEITIVI, P.; LLADO CARBONELL, C. y cols.: "Post-vasectomy semen in 313 males. Statistical analysis, medical aspects, legal implications". Arch. Esp. Urol.; 46: 29-34. 1993.

11. GINGELL, C.; CROSBY, D.; CARROLL, R.: "Review of the complications and medical legal implications of vasectomy". Med. J.; 77: 656-9. 2001

12. GOMEZ DE VICENTE, J. M.; ROMERO CAGIGAL, I.; BLANCO, C. y cols.: "La Historia Natural del aclaramiento de espermatozoides en el semen tras la vasectomía". Actas Urol. Esp.; 28: 286. 2004.

13. SMITH, J.C.; CRANSTON, D.; O'BRIAN, T. y cols.: "Fatherhood without apparent spermatozoa after vasectomy". Lancet; 344: 30. 1994.

14. SCHMIDT, S.S.: "Is timing of post vasectomy- analysis important". Corresp. Br. J Urol; 82: 461. 1998.

15. WHO manual for the standardized investigation, diagnosis and management of the infertile male. Cambrige University Press. 2002.

16. JAFFE, T.M.; KIM, E.D.; HOEKSTRA, T.H. у cols.: "Sperm Pellet analysis: a technique to detect the presence of sperm in men considered to have azoospermia by routine semen analysis". J. Urol.; 159: 1548-50. 1998.

17. ALDERMAN, P.M.: "The lurking sperm: a review of failures in 8879 vasectomies performed by one physician". JAMA; 259:3142. 1988.

18. KENDRICK, J.S.; GINZALES, B.; HUBER, D.H. y cols.: "Complications of vasectomies in the United States". J. Fam. Pract.; 25: 245. 1987.

19. RAJMIL, O.: "Contracepción masculina". In. J Calaf Alsina. Editorial Masson. Manual básico de contracepción. Spain: Barcelona;. 177-184. 1997.

20. ROMERO PEREZ, P.; MERENCIANO CORTINA, F.J.; RAFIE MAZKETLI, W. y cols.: "La vasectomía: estudio de 300 intervenciones. Revision de la literatura nacional y de sus complicaciones". Actas Urol. Esp.; 28: 175. 2004.

21. EDWARDS, I.S.; FARLOW, J.L.: "Nonmotile sperms persisting after vasectomy: do they matter". BMJ; 1: 87. 1979.

22. SCHWINGL, P.J.; GUESS, H.A.: "Safety and effectiveness of vasectomy". Fertil Steril 73:923. 2000. GERLINGER, C.; VANDER MEULEN, E. A.;

**23. DIEBEN, T.O.M. y cols.: "Recommendation for confidence interval and sample size calculation for the Pearl Index". Eur. J. Contracept Reprod Health Care.; 87-92. 2003

24. BENGER, J.R.; SWAMI, S.K.; GINGELL, J.C.: "Persistent spermatozoa after vasectomy: a survey of British urologist". Br. J. Urol.; 76: 376. 1995.

*25. CANOVAS IVORRA, J. A.; TRAMOYERES GALVAN, A.; SANCHEZ BALLESTER, F. y cols.: "Recanalización espontánea tras vasectomía: a propósito de 2 nuevos casos". Arch. Esp. Urol.: 57: 743. 2004.

26. JAMIESON, D.; COSTELLO, J.; TRUSSELL, C. y cols.: "The risk of pregnancy after vasectomy". Obstetrics \& Gynecology.; 103: 848. 2004.

**27. NIVEDITA BATA DHAR, AMIT BHATT, STEPHEN JONES, J.: "Determining the success of vasectomy”. BJU; 97: 773. 2006. 